\title{
In vitro studies on anti-inflammatory activities of kiwifruit peel extract in human THP-1 monocytes
}

\author{
Donatella D’Eliseo $^{\mathrm{a}, \mathrm{b}}$, Elisa Pannucci ${ }^{\mathrm{a}}$, Roberta Bernini ${ }^{\mathrm{a}, *}$, Margherita Campo $^{\mathrm{c}}$, Annalisa Romani ${ }^{\mathrm{c}}$, \\ Luca Santi ${ }^{\mathrm{a}, *}$, Francesca Velotti ${ }^{\mathrm{d}}$ \\ ${ }^{a}$ Department of Agriculture and Forest Sciences (DAFNE), University of Tuscia, Via S. Camillo de Lellis, 01100 Viterbo, Italy \\ ${ }^{\mathrm{b}}$ Department of Experimental Medicine, Sapienza University of Rome, Viale Regina Elena 324, 00161 Rome, Italy \\ c Department of Statistics, Computing, Applications "G. Parenti" (DISIA), Phytolab, University of Florence, Via Ugo Schiff 6, 50019 Florence, Italy \\ ${ }^{\mathrm{d}}$ Department of Ecological and Biological Sciences (DEB), University of Tuscia, Loc. Riello, 01100 Viterbo, Italy
}

\section{A R T I C L E I N F O}

\section{Keywords:}

Kiwifruit (Actinidia deliciosa, cv Hayward) peel extract

Procyanidins

Inflammation

STAT3

Autophagy

\begin{abstract}
A B S T R A C T
Ethnopharmacological relevance: Kiwifruit is native to eastern China and many are the references about the consumption of fruits and fruits extracts of the Actinidia plants in Chinese traditional medicine as therapeutic food supplements to prevent and/or counteract numerous disorders including inflammation-related diseases like cancer.

Aim of the study: Aim of the present work was to obtain a kiwifruit peel extract, rich in polyphenols, and to explore the anti-inflammatory potential by analyzing its capability to target multiple pathways involved in monocyte-mediated inflammatory response.

Materials and methods: The extract was obtained from the fruit peel of Actinidia deliciosa (A.Chev.) C.F.Liang \& A.R.Ferguson, cv Hayward and characterized by HPLC-DAD-ESI-MS. Lipopolysaccharide-stimulated THP-1 monocytes were used as a model of human inflammation in vitro.

Results: Analytical data evidenced that procyanidins resulted the main polyphenols present in the extract, representing the $92 \% \mathrm{w} / \mathrm{w}$ of the total. The extract inhibited the production of inflammatory molecules such as IL6 , IL-1 $\beta$, TNF- $\alpha$ pro-inflammatory cytokines, HMGB1 danger signal and granzyme B serine protease by activated monocytes. In particular, an inhibitory activity of $81 \%, 68 \%, 63 \%, 76 \%$ and $60 \%$ on the extracellular release of IL-6, IL-1 $\beta$, TNF- $\alpha$, HMGB1 and granzyme B, respectively, was observed by western blot analysis. Moreover, the extract prevented STAT3 activation and promoted autophagy.

Conclusions: The reported findings demonstrated a strong and broad anti-inflammatory profile of the kiwifruit peel extract, which makes it a promising preventive and therapeutic natural ingredient for nutraceutical, cosmetic and pharmaceutical formulations to counteract multiple inflammatory disorders.
\end{abstract}

\section{Introduction}

Kiwifruit is the edible berry of the Actinidia plant (Actinidiaceae). The most commonly consumed is the green berry of the Actinidia deliciosa (A.Chev.) C.F.Liang \& A.R.Ferguson, cv Hayward. According to data provided by the Food and Agriculture Organization (FAO) of the United States, kiwifruits cultivation is relevant in China (the native country), Italy, New Zealand, Chile and Greece, which represent the five largest producers in the world.

The use of kiwifruit in Chinese traditional medicine is well documented since the 1400's C.E. while the first recorded reference on the effects against a "cancer-like" disease dates even back to the 720's B.C.E. (Motohashi et al., 2002; Singletary, 2012; Sun et al., 2012). Over the years, kiwifruit has known an ever-wider use in traditional medicine to prevent cardiovascular and degenerative diseases, to relieve disorders caused from dyspepsia, rheumatism, digestive problems and to treat stomach, liver and rectal cancer (Zhi, 1980; Yang, 1981).

More recently, several studies have been focused on the biological activities of the kiwifruit, by individually analyzing extracts derived from either the fruit (Motohashi et al., 2002; Hunter et al., 2011) or the

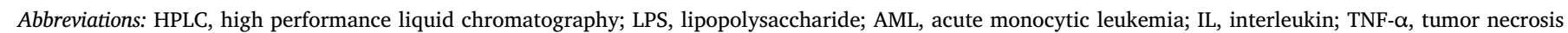

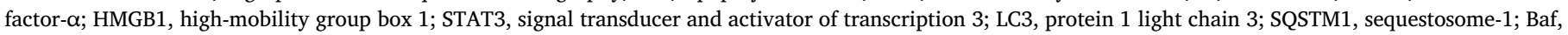
bafilomycin A1; DAMPs, damage associated molecular patterns; 3-MA, 3-methyladenine

* Corresponding authors.

E-mail addresses: roberta.bernini@unitus.it (R. Bernini), luca.santi@unitus.it (L. Santi). 
peel (Motohashi et al., 2001; Yang et al., 2013; Lee et al., 2014). However, to the best of our knowledge, the anti-inflammatory effects of kiwifruit peel extract have never been investigated so far.

Inflammation is the body's normal response to injuries or infections, but, when excessive or persistent, it causes and advances many serious inflammatory diseases including cancer (Lansky and Newman, 2007; Cragg et al., 2009; Martinez-Micaelo et al., 2012; Bernini et al., 2013, 2015). Monocytes and monocyte-derived cells represent critical players in the orchestration of the inflammatory response, representing thus key therapeutic targets for disease treatment. Several studies reported that polyphenols (Andujar et al., 2011; Pietrocola et al., 2012; Bernini et al., 2013, 2015; Rosillo et al., 2014; Liu et al., 2018), and, in particular, procyanidins exerted an anti-inflammatory activity (Bak et al., 2013; De la Iglesia et al., 2010; Martinez-Micaelo et al., 2012).

Based on the consideration that the peel is intimately connected to the pulp, which is the edible part of kiwifruits largely used in Chinese traditional medicine, and the lack of the literature on the biological activities of kiwifruits peel, the aim of this work was to investigate the in vitro anti-inflammatory activity of a peel extract rich in polyphenols obtained from Actinidia deliciosa on activated human THP-1 monocytes, through the analysis of its capability to target multiple processes involved in monocyte-mediated inflammation. Indeed, we investigated the capability of the kiwifruit peel extract to inhibit the production of a number of inflammatory mediators including interleukins (IL-6, IL-1 $\beta$ ) and tumor necrosis factor (TNF- $\alpha$ ) pro-inflammatory cytokines, high mobility group box 1 (HMGB1) danger signal and granzyme B serine protease, to prevent the activation of STAT3 signaling and to promote autophagy.

\section{Material and methods}

\subsection{Plant material}

Low-weight fruits Actinidia deliciosa (A.Chev.) C.F.Liang \& A.R.Ferguson, cv Hayward were collected from Stefano Della Bianca orchard in Cisterna di Latina (Latina, Italy; Latitude: $41^{\circ} 31^{\prime} 42.8^{\prime \prime} \mathrm{N}$; Longitude: $12^{\circ} 47^{\prime} 31.3^{\prime \prime} \mathrm{E}$ ) in July 2016 . A representative of the cultivar has been transplanted in the "Orto dei semplici" section of the "Angelo Rambelli" Botanical Garden of the University of Tuscia, under the accession GS159.

\subsection{Kiwifruit peel extract preparation and characterization}

A kiwifruit peel extract rich in polyphenols was prepared according to a procedure already optimized in our laboratory (Pinelli et al., 2013). Briefly, the peel was isolated from fruits, weighed, frozen in liquid nitrogen and crushed in a mortar. $1 \mathrm{~g}$ of the resulting powder was extracted with $50 \mathrm{~mL}$ of ethanol/ water $=70 / 30 \mathrm{v} / \mathrm{v}$ (water at $\mathrm{pH} 3.2$ ) under magnetic stirring at room temperature for $24 \mathrm{~h}$. After centrifugation of the mixture, the supernatant was recovered and stored at $-20^{\circ} \mathrm{C}$.

The quali-quantitative characterization of polyphenols present in the extract was carried out by HPLC-DAD-ESI-MS using a HP-1100 liquid chromatograph equipped with a DAD detector and a HP 1100 MSD API-electrospray (Agilent Technologies) operating both in negative and positive ionization mode and a column Zorbax SB-Aq $4.6 \mathrm{~mm}$ ID $\times$ $150 \mathrm{~mm}(5 \mu \mathrm{m}$, Agilent) according to already reported in our laboratory (Pinelli et al., 2013). Polyphenols were identified by retention times, spectroscopic and MS data. Procyanidins and hydroxycinnamic acid derivatives were quantified at 280 and $330 \mathrm{~nm}$ using catechin and caffeic acid as reference standard, respectively. The quantitative determination was carried out using five-point regression curves (with $\left.r^{2}>0.9998\right)$. All determinations were carried out in triplicate.

Results are given as means and expressed as mg per gram of peel (Table 1) considering that, each $\mathrm{mL}$ of extract is referred to $0.02 \mathrm{~g}$ of kiwifruit peel; standard error was always $<5 \%$ (Pinelli et al., 2013).
For the biological activities, a stock solution of the extract solubilized in ethanol/water $=70 / 30$, corresponding to a concentration of $12 \mathrm{mg} / \mathrm{mL}$, was expressly prepared as briefly described. From a sample of extract solution, the solvent was removed under reduced pressure by a rotary evaporator (Heidolph, Germany) to afford a powder that was weighted and solubilized with a known volume of ethanol/water $=70$ / 30 to obtain $12 \mathrm{mg} / \mathrm{mL}$ (stock solution). Then, cells were incubated with the indicated concentrations of the extract $(25,50,100$ and $200 \mu \mathrm{g} / \mathrm{mL}$ ), by diluting the stock solution in the cell culture medium. The final concentration of ethanol in the cell culture medium, for both test and corresponding control experiment, was always $<1.6 \%$, compatible with cell viability (assessed by the trypan blue dye exclusion assay) and function (assessed by the analysis of cell proliferation).

\subsection{Cell culture}

THP-1 human monocytic cells, derived from a patient with acute monocytic leukemia (AML), from American Type Culture Collection (ATCC $^{\oplus}$ TIB- $202^{\mathrm{rm}}$; ATCC, MD, USA), were cultured in RPMI 1640 medium (Sigma-Aldrich, St. Louis, MO, USA) containing 10\% FCS (HyClone, Logan, UT, USA), $100 \mu \mathrm{g} / \mathrm{mL}$ of streptomycin and $100 \mathrm{IU} / \mathrm{mL}$ penicillin; they were maintained in a $5 \% \mathrm{CO}_{2}$ incubator at $37^{\circ} \mathrm{C}$. Cells were mycoplasma free (EZ-PCR Mycoplasma test kit; Biological Industries, Cromwell, CT, USA).

\subsection{Cell treatments}

For THP-1 activation, cells were cultured with $100 \mathrm{ng} / \mathrm{mL}$ lipopolysaccharide (LPS) from Escherichia coli 055:B5 (L2880 Sigma) for $24 \mathrm{~h}$. THP-1 cells were incubated with ethanolic solution alone (Control) or with the extract, solubilized in ethanolic solution, at the indicated doses, for the indicated times. For the autophagic investigation, THP-1 cells were cultured with the extract $(50 \mu \mathrm{g} / \mathrm{mL})$ for $24 \mathrm{~h}$ and then treated with Bafilomycin A1 (Baf) $(20 \mathrm{nM})$ (Santa Cruz Biotechnology Inc., Santa Cruz, CA, USA), an inhibitor of vacuolar-H +-ATPase, for the last $2 \mathrm{~h}$. In some experiments, 3-methyladenine (3MA) ( $0.2 \mathrm{mM}$ ) (Santa Cruz Biotechnology Inc.) was added $2 \mathrm{~h}$ before the extract treatment.

\subsection{Antibodies}

The following primary antibodies were used: rabbit polyclonal antiIL-6 (Gene Tex, Alton Pkwy Irvine, CA), rabbit polyclonal anti-IL-1 $\beta$ (Gene Tex, CA, USA), mouse monoclonal anti-TNFa (Santa Cruz), rabbit polyclonal anti-HMGB1 (Abcam, Cambridge, UK), mouse monoclonal anti-granzyme B (Calbiochem, San Diego, CA, USA), mouse monoclonal anti-phospho-STAT3 (pY705) (BD Transduction Laboratories, San Jose, CA, USA), mouse monoclonal anti-STAT3 (BD Transduction Laboratories), rabbit polyclonal anti-LC3 (Novus Biologicals, Littleton, CO, USA), mouse monoclonal anti-p62 (BD Transduction Laboratories) and mouse monoclonal anti- $\beta$-actin Ac-40 (Sigma-Aldrich).

\subsection{Cell viability and proliferation assay}

After each treatment, cell viability was assessed by the trypan blue dye exclusion assay, as previously reported (D'Eliseo et al., 2017). The analysis of cell proliferation was performed using $0.05 \%$ trypan blue solution to count the number of live cells within a Neubauer chamber. At least three replicate counts were conducted by the same operator at each time.

\subsection{Cytokine measurement}

Cytokines were measured in conditioned media using the MILLIPLEX $^{\circ}$ MAP Human Cytokine/Chemokine Magnetic Bead panel 
Table 1

Quali-quantitative analysis of phenolic compounds found in the kiwifruit peel extract.

\begin{tabular}{|c|c|c|c|c|c|}
\hline Compound & $t_{R}(\min )$ & MW & Negative ions $(m / z)^{a}$ & $\lambda_{\max }, \lambda_{\min }$ & $\mathrm{mg} / \mathrm{g}^{\mathrm{b}}$ \\
\hline 6-Hydroxy-7-( $\beta$-D-glucopiranolyloxy coumarin & 10.9 & 340 & 339,177 & & Traces \\
\hline Caffeic acid derivative & 14.0 & 356 & $355,297,239,179,161,135$ & 326,264 & $0.243 \pm 0.007$ \\
\hline Ferulic acid glucoside & 16.2 & 356 & 355,193 & 312,255 & $0.174 \pm 0.006$ \\
\hline Ferulic acid dehydrodimer & 17.5 & 370 & $369,193,179,135$ & 324,265 & $0.042 \pm 0.002$ \\
\hline Dimethyl caffeic acid hexoside & 16.9 & 370 & $369,207,191$ & & Traces \\
\hline Procyanidin trimer & 17.8 & 866 & $865,577,289$ & 280,258 & $1.193 \pm 0.042$ \\
\hline Procyanidin trimer & 20.2 & 866 & $865,577,289$ & 280,258 & $0.659 \pm 0.025$ \\
\hline Procyanidin trimer & 21.8 & 866 & $865,577,289$ & 280,258 & $0.665 \pm 0.019$ \\
\hline Procyanidin tetramer & 24.2 & 1154 & $1153,865,577,289$ & 280,258 & $0.142 \pm 0.003$ \\
\hline Procyanidin trimer & 26.1 & 866 & $865,577,289$ & 280,258 & $0.880 \pm 0.035$ \\
\hline Procyanidin trimer & 27.1 & 866 & $865,577,289$ & 280,258 & $1.187 \pm 0.047$ \\
\hline Procyanidin dimer & 39.9 & 578 & 577,289 & 280,258 & $0.696 \pm 0.038$ \\
\hline Total hydroxycinnamic derivatives & & & & & $0.459 \pm 0.015$ \\
\hline Total procyanidins & & & & & $5.422 \pm 0.209$ \\
\hline Total polyphenols & & & & & $5.881 \pm 0.224$ \\
\hline
\end{tabular}

a $\mathrm{m} / \mathrm{z}$ of the most abundant ESI/MS signals (fragmentor: $120 \mathrm{eV}$ ).

b $\mathrm{mg}$ of phenolic compound per gram of peel.

(Millipore, Billerica, MA, USA) according to the manufacturer's instructions and detected by Bio-Plex ${ }^{\circ}$ MAGPIX $^{\mathrm{TM}}$ Multiplex Reader (Biorad).

\subsection{Western blot analysis}

Cells were washed twice in PBS and cell lysates were prepared by a solution containing $50 \mathrm{mM}$ TRIS- $\mathrm{HCl} \mathrm{pH} 7.6,150 \mathrm{mM} \mathrm{NaCl}, 0.5 \%$ TRITON X-100, 0.5\% Sodium deoxycolate, $0.1 \%$ SDS and the protease inhibitor mixture "Complete" (Roche Diagnostic GmbH, Mannheim, Germany). For the experiments analyzing the extracellular release of inflammatory mediators, the conditioned media was collected. Proteins were separated by SDS-PAGE and blotted onto nitrocellulose membranes (Whatman-Protan, Sigma-Aldrich). The membranes were blocked with 5\% Non-Fat Dry Milk (Bio-Rad, Hercules, CA, USA), probed with specific primary antibodies overnight, at $4{ }^{\circ} \mathrm{C}$, washed and incubated with appropriated secondary antibodies. The reaction was revealed by horseradish peroxidase (HRP)-coupled secondary reagents (Bio-Rad) and developed by enhanced chemiluminescence (Amersham ECL Western Blotting Detection Reagent, GE Healthcare, MI, Italy).

\subsection{Densitometric analysis}

The quantification of protein bands was performed by densitometric analysis using Quantity One 1-D analysis software (Bio-Rad) and band intensities (b.i., band volume/area x mean pixel intensity), normalized for $\beta$-actin, as previously reported (D'Eliseo et al., 2017).

\subsection{Statistical analysis}

Student's $t$-test was applied for all analyses; $p<0.05$ was considered significant. All experiments were performed at least three times.

\section{Results and discussion}

\subsection{Kiwifruit peel extract preparation and characterization}

The peel of the green berry of Actinidia deliciosa (A.Chev.) C.F.Liang \& A.R.Ferguson, cv Hayward was treated with ethanol/acidified water $(\mathrm{pH} 3.2)=70 / 30 \mathrm{v} / \mathrm{v}$ according to a procedure already optimized in our laboratory (Pinelli et al., 2013) to obtain a polyphenolic-rich extract. The quali-quantitative analysis of the extract was performed by HPLC-DAD-ESI-MS. As reported in Table 1, procyanidins dimers, trimers and tetramers resulted the main class of polyphenols present in the extract with $5.422 \pm 0.209 \mathrm{mg}$ per gram of peel, representing the
$92 \% \mathrm{w} / \mathrm{w}$ of the total; hydroxycinnamic acid derivatives covered the remaining percentage $(8 \% \mathrm{w} / \mathrm{w})$ with $0.459 \pm 0.015 \mathrm{mg} / \mathrm{g}$.

\subsection{Effects of kiwifruit peel extract on THP-1 cell viability and proliferation}

Kiwifruit peel extract was tested for cytotoxicity in THP-1 cells by culturing them in the presence of increasing extract concentrations (from 12.5 to $200 \mu \mathrm{g} / \mathrm{mL}$ ) for $24 \mathrm{~h}$. The trypan-blue dye exclusion assay showed that the extract did not affect the viability of THP-1 cells at none of the concentrations tested (Fig. 1A). Moreover, the evaluation of cell growth showed that the cell number was not affected up to an extract concentration of $100 \mu \mathrm{g} / \mathrm{mL}$ and then decreased in a dose dependent manner (Fig. 1B), suggesting that a proliferative arrest might occur at high concentrations. Thus, all subsequent experiments were performed treating THP-1 cells with a dose of $50 \mu \mathrm{g} / \mathrm{mL}$ for $24 \mathrm{~h}$.

(a)

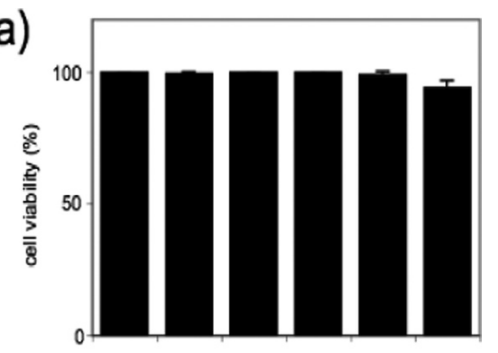

(b)

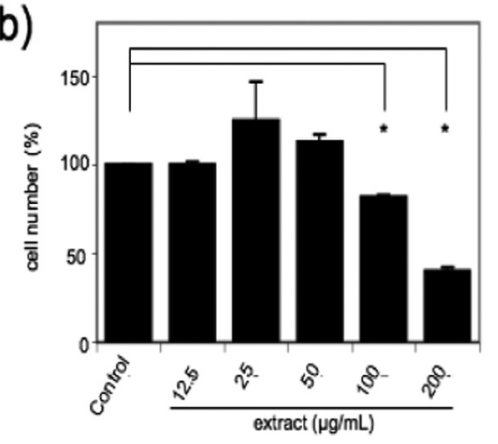

Fig. 1. Effects of kiwifruit peel extract on THP-1 cell viability (a) and proliferation (b) at $24 \mathrm{~h}$ of treatment. Data show mean of the percentage (\%) plus S.D. of three independent experiments; ${ }^{*} p<0.005$. 
(a)

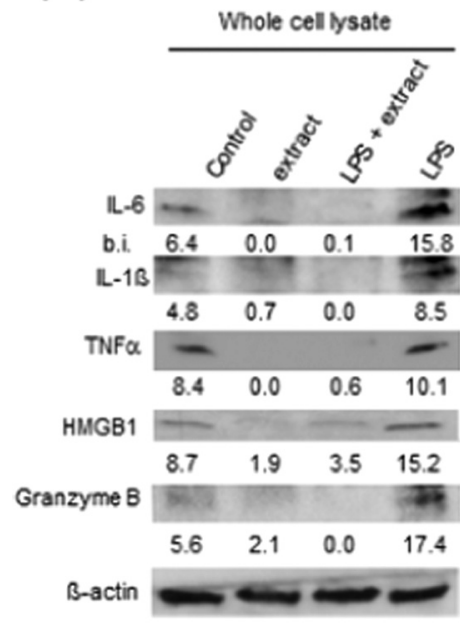

(b)

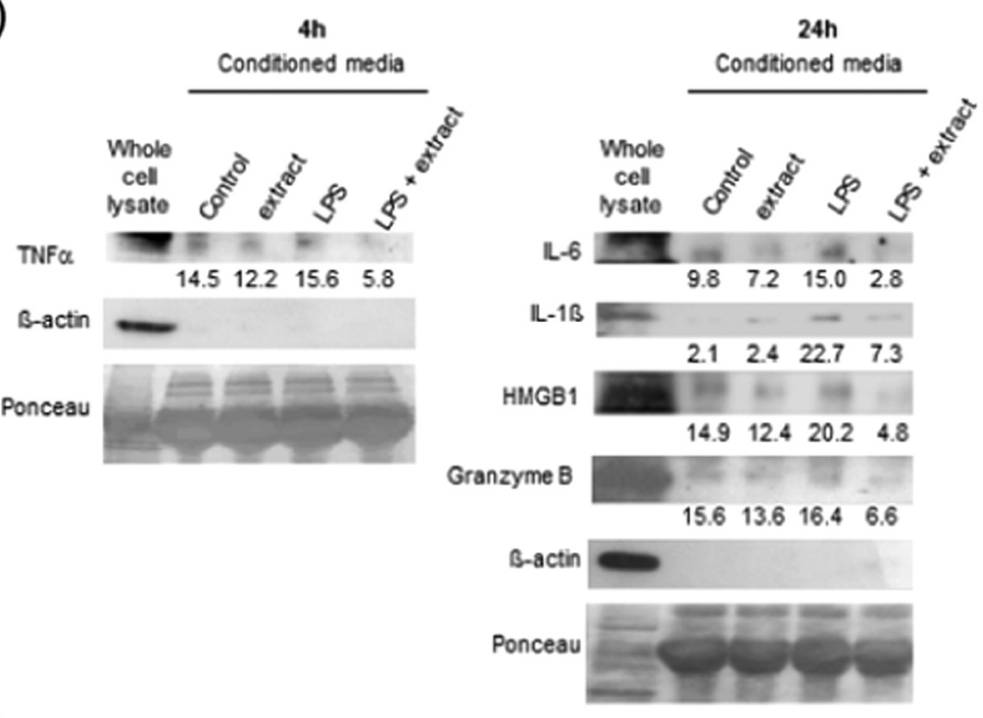

(c)
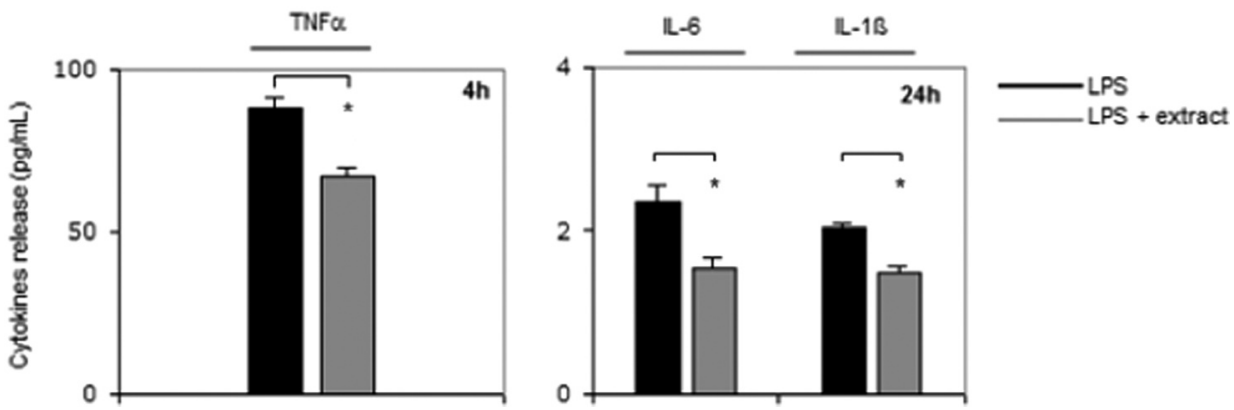

Fig. 2. Effects of kiwifruit peel extract on the intracellular content (a) and the extracellular release (b, c) of inflammatory mediators by THP-1 cells. Data show Western blot on cell lysates (a) and conditioned media (b); $\beta$-Actin and Ponceau staining served as intracellular and extracellular loading control, respectively; numbers indicate band intensities (b.i.) = band volume/area $\mathrm{x}$ mean pixel intensity, normalized for $\beta$-actin or Ponceau staining and quantified using Quantity One 1D analysis software; representative experiments out of three. Histograms represent the mean plus S.D. of cytokine concentrations of three independent experiments, evaluated in conditioned media by Luminex multiplex assay, (c); * $p<0.005$.

\subsection{Kiwifruit peel extract inhibited LPS-inducible and constitutive IL-6, IL- $1 \beta$ and TNF- $\alpha$ production}

Pro-inflammatory cytokines IL- 6 , IL- $1 \beta$ and TNF- $\alpha$ play a pivotal role in the progression of the inflammatory process as a result of monocyte activation. We, therefore, investigated the capability of the extract to affect the production of the three cytokines by activated monocytes. To this purpose, LPS-stimulated and unstimulated (Control) THP-1 cells were treated with the extract and the protein levels of IL-6, IL-1 $\beta$ and TNF- $\alpha$ were measured by Western blot analysis and normalized for $\beta$-actin or Ponceau stain. Since it has been previously reported that the maximum of TNF- $\alpha$ release is at earlier times compared to IL- 6 and IL- $1 \beta$, we analyzed the release of TNF- $\alpha$ at $4 \mathrm{~h}$, whereas IL- 6 and IL- $1 \beta$ releases were measured at $24 \mathrm{~h}$ after treatment. Basal levels of both intracellular and extracellular cytokines were detected in unstimulated THP-1 cells, and, as expected, they increased when THP-1 cells were stimulated with LPS (100 ng/mL) (Fig. 2A and B). However, when the cell cultures were treated with the extract, an almost complete reduction of the intracellular content (Fig. 2A) associated to a significant decrease of the release (Fig. 2B) of all the three cytokines was observed in both LPS-stimulated and unstimulated THP-1 cells. Moreover, the inhibition of monocyte-mediated release of cytokines by the extract was confirmed by Luminex multiplex assay (Fig. 2C). These results showed that the kiwifruit peel extract suppressed the production of pro-inflammatory cytokines, indicating a potential anti-inflammatory activity by the extract on inflammatory monocytes.

\subsection{Kiwifruit peel extract inhibited LPS-inducible and constitutive HMGB1 production}

Extracellular HMGB1 release by the inflammatory microenvironment is involved in the pathogenesis of multiple human inflammatory diseases (e.g., trauma, ischemia, chronic inflammatory disorders, autoimmune diseases and cancer), representing a key molecular target in these diseases (Bianchi and Manfredi, 2007). Thus, we investigated the capability of the extract to modulate HMGB1 production by activated monocytes. We found that both the intracellular content (Fig. 2A) and the extracellular release (Fig. 2B, right panel) of HMGB1 were significantly inhibited by the extract in both LPS-stimulated and unstimulated THP-1 cells. This finding further supports the potential of this extract for anti-inflammatory activity and, to the best of our knowledge, this activity has never been described before in studies investigating the anti-inflammatory capability of kiwifruits.

\subsection{Kiwifruit peel extract inhibited LPS-inducible and constitutive granzyme B production}

The serine protease granzyme $\mathrm{B}$ is implicated in extracellular functions involved in inflammation, cytokine activation and autoimmunity, and elevated levels of soluble granzyme B have been reported in plasma of patients with inflammatory diseases (Hiebert and Granville, 2012). To notice, it has been demonstrated that monocytes/ macrophages express granzyme B in the lesion areas of inflammatory 
diseases, including atherosclerosis and rheumatoid arthritis. Thus, targeting granzyme B provides new pharmaceutical agents for inflammatory disorders. Therefore, we analyzed whether the extract could affect the production of granzyme B by THP-1 cells. We found that the extract significantly inhibited both the intracellular expression (Fig. 2A) and the extracellular release (Fig. 2B, right panel) of granzyme B by LPS-stimulated and unstimulated THP-1 cells. This is the very first time that a kiwifruit extract has been reported to mediate this activity. This finding further supports the interest in this extract for its antiinflammatory potential.

\subsection{Kiwifruit peel extract prevented STAT3 activation}

STAT3 activation by pathogens, growth factors or cytokines, such as IL-6, induces the production of pro-inflammatory cytokines and regulates diverse inflammatory processes, cell proliferation and survival (Yu et al., 2009). In particular, STAT3 activation in monocytes contributes to neovascular age-related macular degeneration (Chen et al., 2016), as well as, promotes liver tumorigenesis (Wu et al., 2011). Indeed, STAT3 in inflammatory monocytes appears a promising target for pharmacological intervention in the prevention and treatment of the development of inflammatory diseases and cancer (Yu et al., 2009). Thus, we examined the capability of the extract to modulate STAT3 activation, by analyzing its phosphorylation at Tyr 705. As expected, STAT3 phosphorylation (p-STAT3) levels significantly increased when THP-1 cells were exposed to LPS (Fig. 3). However, treatment with the extract significantly inhibited p-STAT3 in LPS-stimulated as well as in unstimulated THP-1 cells (Fig. 3). Moreover, considering that STAT3 signaling is triggered by IL- 6 upon interaction with IL- 6 receptor, we can suppose that a correlation exists between the inhibition of IL-6 production (Fig. 2) and the inhibition of STAT3 activation (Fig. 3). Inhibition of IL- 6 production and STAT3 activation by the extract are both relevant findings, since numerous studies demonstrated the association of down-regulation of IL-6 and/or IL-6 signaling (i.e., STAT3) with therapeutic results in inflammatory diseases. In addition, an important role of STAT3 has been identified in the development of different cancers, including AML (Redell et al., 2011). Therefore, our result, showing the inhibition of the constitutive level of STAT3 in unstimulated THP-1 monocytic leukemia cells, suggests that the extract might also exert anti-cancer activity in AML. Considering all these findings, we think that the extract might represent a potential promising candidate to target the IL-6/STAT3 pathway in inflammatory diseases and cancer.

\subsection{Kiwifruit peel extract promoted autophagy}

Autophagy mediates the clearance of cytoplasmic molecules, organelles and pathogens, and, interestingly, orchestrates inflammation,

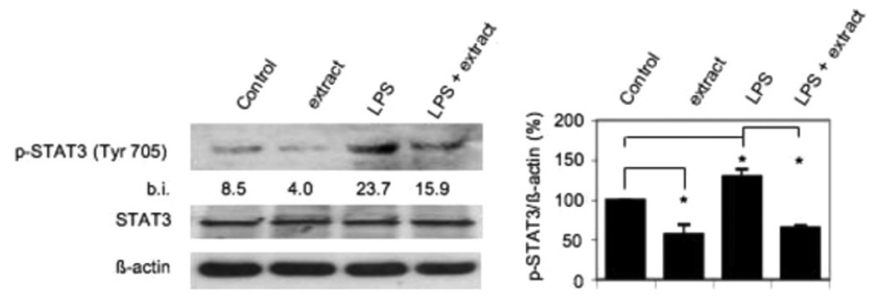

Fig. 3. Effect of kiwifruit peel extract on STAT3 activation in THP-1 cells. Data show STAT3 Tyr705 phosphorylation (p-STAT3), evaluated by western blot (left panel); total STAT3 and $\beta$-actin served as controls; numbers indicate band intensities (b.i.) = band volume/area $\mathrm{x}$ mean pixel intensity, normalized for $\beta$ actin and quantified using Quantity One 1-D analysis software; representative experiment out of three. Histograms represent the percentage (\%), respect to the control, of the mean plus S.D. of the densitometric analysis of p-STAT3/ $\beta$ actin ratio of three different experiments (right panel); ${ }^{*} p<0.005$.
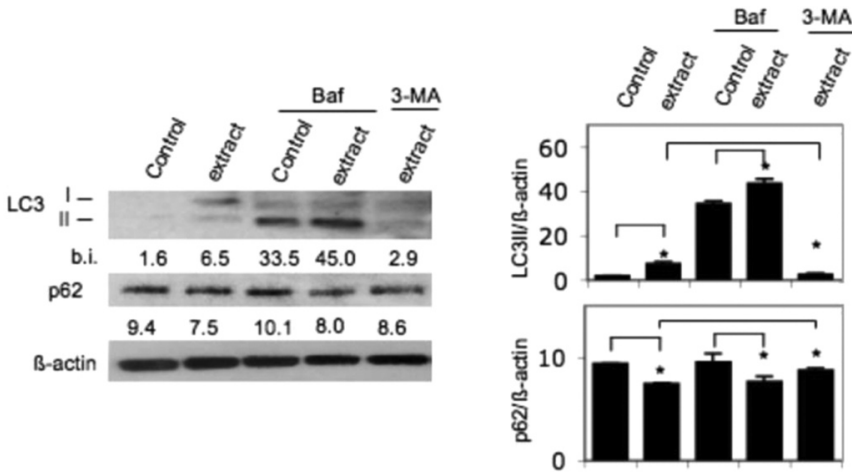

Fig. 4. Effect of kiwifruit peel extract on autophagy in THP-1 cells. Data show the expression of LC3I/II and SQSTM1/p62 autophagic markers, analyzed by western blot (left panel); $\beta$-actin was included as loading control; numbers indicate band intensities (b.i.) = band volume/area $\mathrm{x}$ mean pixel intensity, normalized for $\beta$-actin and quantified using Quantity One 1-D analysis software; representative experiment out of three. Histograms represent the mean plus S.D. of the densitometric analysis of LC3II/ $\beta$-actin and p62/ $\beta$-actin ratio of three independent experiments (right panel); ${ }^{*} p<0.005$.

immunity and cancer (Levine et al., 2011). In particular, activation of autophagy in monocytes exerts anti-inflammatory activity, affecting inflammatory cytokine production by different mechanisms, including the inhibition of inflammasomes, leading to the suppression of IL- $1 \beta$ and IL-18 maturation. Therefore, we examined whether the kiwifruit peel extract could affect autophagy in THP-1 cells. To investigate the autophagic process, two main autophagic markers such as the microtubule-associated protein 1 light chain 3 (LC3) and the sequestosome-1 (SQSTM1)/ubiquitin-binding protein p62 were evaluated by Western blot (Klionsky, 2016; D'Eliseo et al., 2017). During autophagy, LC3 is processed post-translationally into soluble LC3-I and, in turn, converted to membrane-bound LC3-II that correlates with the extent of autophagosomes. Therefore, we identified LC3 in the LC3-II form in THP-1 cells and, as shown in Fig. 4, LC3II formation significantly increased after treatment with the extract, suggesting induction of autophagy. Moreover, we observed a further accumulation of LC3-II when extracttreated cells were cultured in the presence of the vacuolar $\mathrm{H}+$-ATPase inhibitor bafilomycin A1 (Baf), confirming the induction of a complete autophagic flux by the extract (Fig. 4). Furthermore, the addition of 3MA autophagic inhibitor to extract-treated THP-1 cells decreased LC3II formation, confirming thus that LC3II protein levels were the result of the autophagic process (Fig. 4). Then, we analyzed the other autophagic marker such as SQSTM1/p62, which, being part of the assembled autophagosome subsequently degraded in autolysosomes, serves as an index of autophagic degradation. As shown in Fig. 4, SQSTM1/p62 levels decresed in THP-1 cells treated with the extract, further indicating that the extract was able to promote a complete autophagic process. This is a very significant finding, since defects in autophagy have been linked to a wide range of diseases (e.g., cardiomyopathy, neurodegenerative disorders, cancers), and compounds capable of inducing autophagy have been recently received attention for the treatment of inflammatory diseases (Levine et al., 2011).

\section{Conclusions}

In summary, this study has proved for the first time that an extract rich in procyanidins, obtained from the peel of kiwifruits, is a suppressor of the production of a number of inflammatory mediators such as pro-inflammatory cytokines, HMGB1, granzyme B and STAT3 and a promoter of autophagy on activated human THP-1 monocytes. These results are consistent with reports demonstrating that polyphenols from different origin are endowed with the inhibition of pro-inflammatory cytokine production (Rosillo et al., 2014), the suppression of STAT3 
activation (Andujar et al., 2011) and the promotion of autophagy (Pietrocola et al., 2012). All the data show that the extract may represent a potential promising anti-inflammatory natural ingredient for pharmaceutical and nutraceutical formulations. However, this assumption requires further verification using in vivo models.

\section{Acknowledgments}

The authors are grateful to Stefano Della Bianca for the availability to furnish kiwifruits and technical support and to Monica Fonck of the "Angelo Rambelli" Botanical Garden (University of Tuscia) for her availability and expertise. The authors gratefully acknowledge MIUR (MInistry for education, University and Research) for the financial support (Law 232/216, Department of Excellence) of a part of this research.

\section{Conflict of interest}

No conflict of interests for all authors.

\section{Author contribution}

Donatella D'Eliseo and Elisa Pannucci were involved in experimental execution and biological data collection. Margherita Campo carried the chromatographic analysis of the kiwifruit peel extract. Roberta Bernini, Annalisa Romani, Luca Santi and Francesca Velotti were involved in experimental design, in data analysis and interpretation, in manuscript writing and editing.

\section{References}

Andujar, I., Recio, M.C., Giner, R.M., Cienfuegos-Jovellanos, E., Laghi, S., Muguerza, B., Rios, J.L., 2011. Inhibition of ulcerative colitis in mice after oral administration of a polyphenol-enriched cocoa extract is mediated by the inhibition of STAT1 and STAT3 phosphorylation in colon cells. J. Agric. Food Chem. 59, 6474-6483.

Bak, M.-J., Truong, V.L., Kang, H.S., Jun, M., Jeong, W.S., 2013. Anti-inflammatory effect of procyanidins from wild grape (Vitis amurensis) seeds in LPS-induced RAW 264.7 cells. Oxid. Med. Cell. Longev. https://doi.org/10.1155/2013/40932.

Bernini, R., Merendino, N., Romani, A., Velotti, F., 2013. Naturally occurring hydroxytyrosol: synthesis and anticancer potential. Curr. Med. Chem. 20, 655-670.

Bernini, R., Gilardini Montani, M.S., Merendino, N., Romani, A., Velotti, F., 2015. Hydroxytyrosol-derived compounds: a basis for the creation of new pharmacological agents for cancer prevention and therapy. J. Med. Chem. 58, 9089-9107.

Bianchi, M.E., Manfredi, A.A., 2007. High-mobility group box 1 (HMGB1) protein the crossroads between innate and adaptive immunity. Immunol. Rev. 220, 35-46.

Chen, M., Lechner, J., Zhao, J., Toth, L., Hogg, R., Silvestri, G., Kissenpfennig, A., Chakravarthy, U., Xu, H., 2016. STAT3 Activation in circulating monocytes contributes to neovascular age-related macular degeneration. Curr. Mol. Med. 16, 412-423.

Cragg, G.M., Grothaus, P.G., Newman, D.J., 2009. Impact of natural products on developing new anti-cancer agents. Chem. Rev. 109, 3012-3043.

D'Eliseo, D., Di Renzo, L., Santoni, A., Velotti, F., 2017. Docosahexaenoic acid (DHA) promotes immunogenic apoptosis in human multiple myeloma cells, induces autophagy and inhibits STAT3 in both tumor and dendritic cells. Genes Cancer 8, 426-437.

De la Iglesia, R., Milagro, F.I., Campion, J., Boque, N., Martinez, J.A., 2010. Healthy properties of proanthocyanidins. Biofactors 36, 159-168.

Hiebert, P.R., Granville, D.J., 2012. Granzyme B in injury, inflammation, and repair. Trends Mol. Med. 18, 732-741.

Hunter, D.C., Greenwood, J., Zhang, J., Skinner, M.A., 2011. Antioxidant and "natural protective" properties of kiwifruit. Curr. Top. Med. Chem. 11, 1811-1820.

Klionsky, D.J., 2016. Guidelines for the use and interpretation of assays for monitoring autophagy (3rd edition). Autophagy 12, 1-222.

Lansky, E.P., Newman, R.A., 2007. Punica granatum (pomegranate) and its potential for prevention and treatment of inflammation and cancer. J. Ethnopharmacol. 109, 177-209.

Lee, C.C., Lee, B.H., Wu, S.C., 2014. Lanskpeel (kiwi fruits) ethanol extracts protected neural cells apoptosis induced by methylglyoxal through Nrf2 activation. Pharm. Biol. 52, 628-636.

Levine, B., Mizushima, N., Virgin, H.W., 2011. Autophagy in immunity and inflammation. Nature 469, 323-335.

Liu, H., Ma, S., Xia, H., Lou, H., Zhu, F., Sun, L., 2018. Anti-inflammatory activities and potential mechanisms of phenolic acids isolated from Salvia miltiorrhiza f. alba roots in THP-1 macrophages. J. Ethnopharmacol. 222, 201-207.

Martinez-Micaelo, N., Gonzalez-Abuin, N., Ardevol, A., Pinent, M., Blay, M.T., 2012. Procyanidins and inflammation: molecular targets and health implications. BioFactors 257-265.

Motohashi, N., Shirataki, Y., Kawasw, M., Tani, S., Sakagami, H., Satoh, K., Kurihara, T., Nakashima, H., Wolfard, K., Miskolci, C., Molnar, J., 2001. Biological activity of kiwifruit peel extracts. Phytother. Res. 15, 337-343.

Motohashi, N., Shirataki, Y., Kawase, M., Tani, S., Sakagami, H., Satoh, K., Kurihara, T., Nakashima, H., Muacis, I., Varga, A., Molnar, J., 2002. Cancer prevention and therapy with kiwifruit in Chinese folklore medicine: a study of kiwifruit extracts. J. Ethnopharmacol. 81, 357-364.

Pietrocola, F., Marino, G., Lissa, D., Vacchelli, E., Malik, S.A., Niso-Santano, M., Zamzami, N., Galluzzi, L., Maiuri, M.C., Kroemer, G., 2012. Pro-autophagic polyphenols reduce the acetylation of cytoplasmic proteins. Cell Cycle 11, 3851-3860.

Pinelli, P., Romani, A., Fierini, E., Remorini, D., Agati, G., 2013. Characterisation of the polyphenol content in the kiwifruit (Actinidia deliciosa) exocarp for the calibration of a fruit-sorting optical sensor. Phytochem. Anal. 24, 460-466.

Redell, M.S., Ruiz, M.J., Alonzo, T.A., Gerbing, R.B., Tweardy, D.J., 2011. STAT3 signaling in acute myeloid leukemia: ligand-dependent and -independent activation and induction of apoptosis by a novel small-molecule Stat3 inhibitor. Blood 117, 5701-5709.

Rosillo, M.A., Alcaraz, M.J., Sanchez-Hidalgo, M., Fernandez-Bolanos, J.G., Alarcon-dela-Lastra, C., Ferrandiz, M.L., 2014. Anti-inflammatory and joint protective effects of extra-virgin olive-oil polyphenol extract in experimental arthritis. J. Nutr. Biochem. $25,1275-1281$.

Singletary, K., 2012. Kiwifruit. Overview of potential health benefits. Nutr. Today 47, $133-147$.

Sun, S., Xu, H., Ngeh, L., 2012. The evaluation of Chinese therapeutic food per the treatment of moderate disylipidemia. Evid.-Based Complement. Altern. Med. 1-11 (and references therein).

Wu, W.-Y., Li, J., Wu, Z.-S., Zhang, C.-L., Meng, X.L., 2011. STAT3 activation in monocytes accelerates liver cancer progression. BMC Cancer 11, 506.

Yang, J.-X., 1981. Chinese Pharmaceuticals for Cancers. General people's health publishers, Peking, pp. 121-122.

Yang, H., Lee, Y.-C., Han, K.-S., Singh, H., Yoon, M., Park, J.-H., Cho, C.-W., Cho, S., 2013. Green and gold kiwifruit peel ethanol extracts potentiate pentobarbital-induced sleep in mice via a GABAergic mechanism. Food Chem. 136, 160-163.

Yu, H., Pardoll, D., Jove, R., 2009. STATs in cancer inflammation and immunity: a leading role for STAT3. Nat. Rev. Cancer 9, 798-809.

Zhi, C.-J., 1980. Chinese Anti-Cancer Agents. Hua-lian Publishers, Taipei, pp. 74-75. 